Supporting Information

\title{
Real-Time Detection of Glyphosate by a Water-Gated Organic Field-Effect Transistor with a Microfluidic Chamber
}

\author{
Koichiro Asano, ${ }^{\dagger}$ Pierre Didier,${ }^{\dagger}$, Kohei Ohshiro $^{\dagger}{ }^{\dagger}$ Nicolas Lobato-Dauzier, ${ }^{\dagger}, \star$ Anthony J.

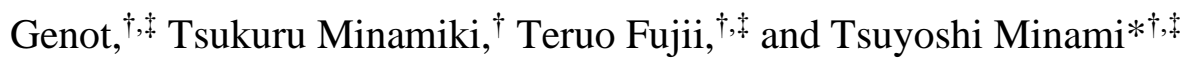 \\ †Institute of Industrial Science, The University of Tokyo, 4-6-1 Komaba, Meguro-ku, Tokyo, \\ 153-8505, Japan.
}

LIMMS/CNRS-IIS (UMI2820), The University of Tokyo, 4-6-1 Komaba, Meguro-ku, Tokyo, 153-8505, Japan.

*E-Mail:tminami@iis.u-tokyo.ac.jp

Tel: $+81-354526364$

Number of pages: 6

Number of figures: 8

Number of tables: 3

\section{CONTENTS}

$\begin{array}{ll}\text { 1. Fabrication scheme of the microfluidic chamber } & \text { S2 }\end{array}$

$\begin{array}{ll}\text { 2. Fabrication scheme of the WG-OFET } & \text { S2 }\end{array}$

3. Basic characteristics of the fabricated WG-OFET $\quad$ S3

4. FM-AFM images of the surface of the P3CPT $\quad$ S4

5. The WG-OFET characteristics related with the sensing application $\quad$ S4 


\section{Fabrication scheme of the microfluidic chamber}

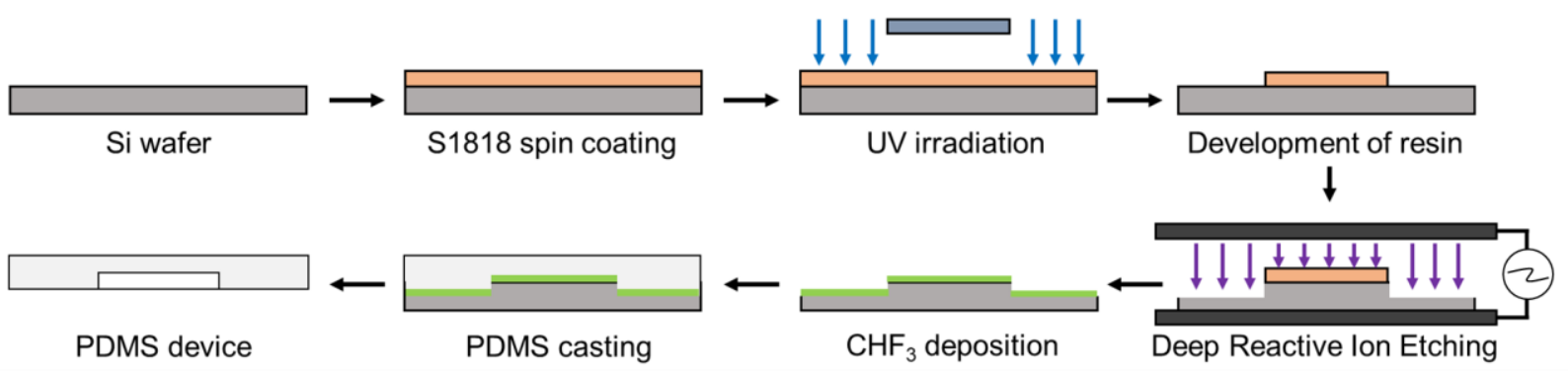

Figure S1. The scheme of the fabrication process of the microfluidic chamber.

\section{Fabrication scheme of the WG-OFET}

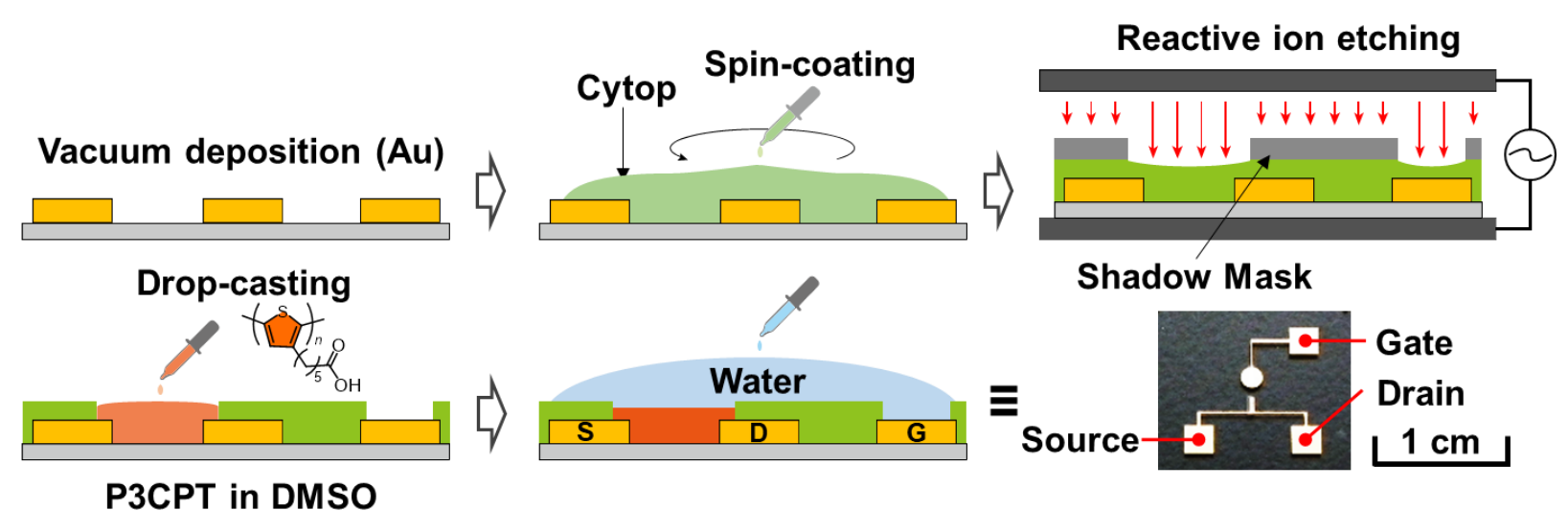

Figure S2. The scheme of the fabrication process of the WG-OFET based sensor.

\section{Basic characteristics of the fabricated WG-OFET}

The transfer and output characteristics of the fabricated WG-OFET were measured with the flow of $100 \mathrm{mM}$ HEPES buffer solution containing $100 \mathrm{mM} \mathrm{NaCl}$. The OFET characteristics were measured at the constant drain-source voltage $\left(V_{\mathrm{DS}}\right)$ and gate-source voltage $\left(V_{\mathrm{GS}}\right)$ of $-0.3 \mathrm{~V}$. The flow rate was set at $10 \mu \mathrm{L} \mathrm{min}{ }^{-1}$. Because the electric double layer capacitance (EDLC) induces 
an ultimately strong electric field in comparison with the solid-state capacitances, the mobility and the contact resistance could not be estimated.

(A)

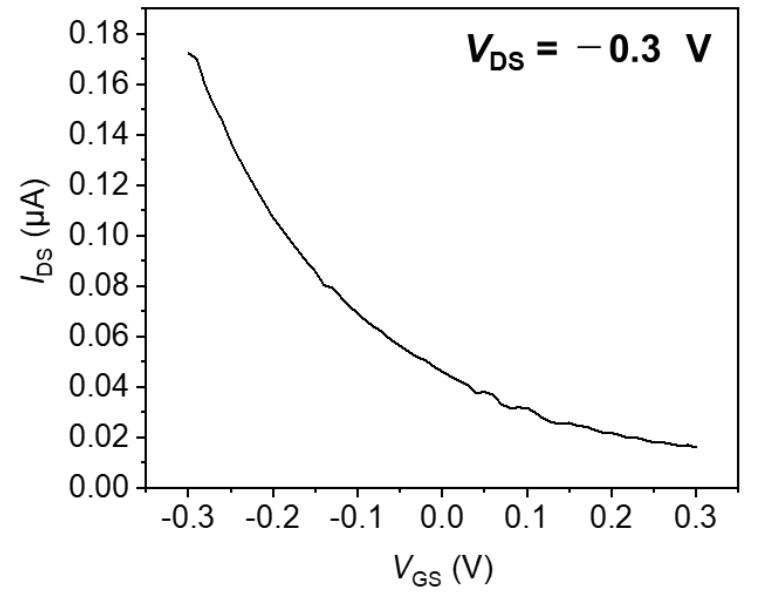

(B)

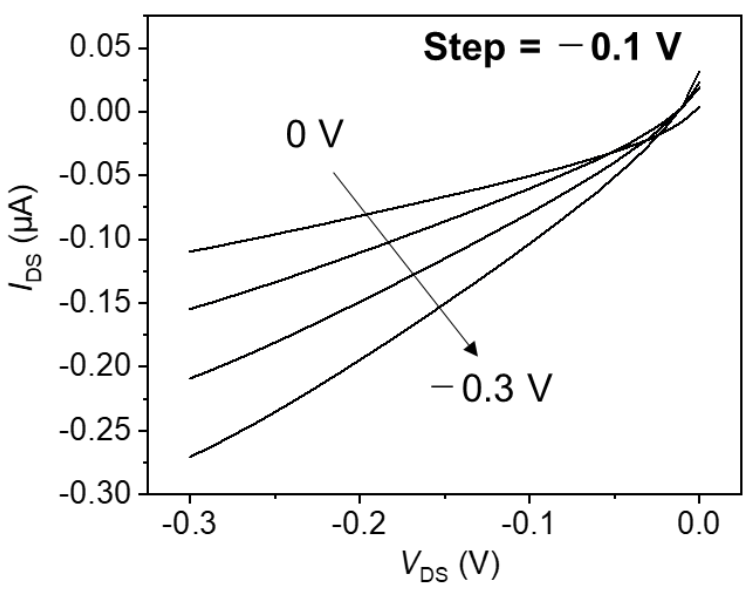

Figure S3. The basic characteristics of the fabricated WG-OFET integrated with the microfluidic chamber. A) Transfer and B) output characteristics with the injection of the HEPES (100 mM) buffer with $\mathrm{NaCl}(100$ $\mathrm{mM})$ at flow rate $10 \mu \mathrm{L} \mathrm{min}^{-1}$. The inconsistency between the transfer and output characteristics (at $V_{\mathrm{Ds}}=$ $V_{\mathrm{GS}}=-0.3 \mathrm{~V}$ ) is probably due to the difference in the charge accumulation at the OSC/electrolyte interface triggered by the gate bias.

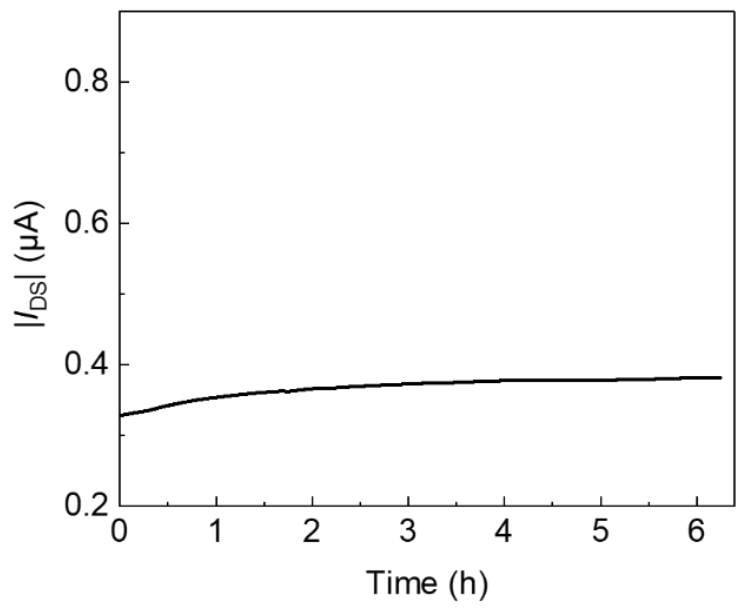

Figure S4. The time-dependent change of $I_{\mathrm{DS}}$ of the microfluidic-attached WG-OFET with continuous flow $\left(10 \mu \mathrm{L} \mathrm{min}^{-1}\right)$ of the HEPES buffer solution $(100 \mathrm{mM})$ containing $\mathrm{NaCl}(100 \mathrm{mM})$. 


\section{FM-AFM images of the surface of the P3CPT}

(A)

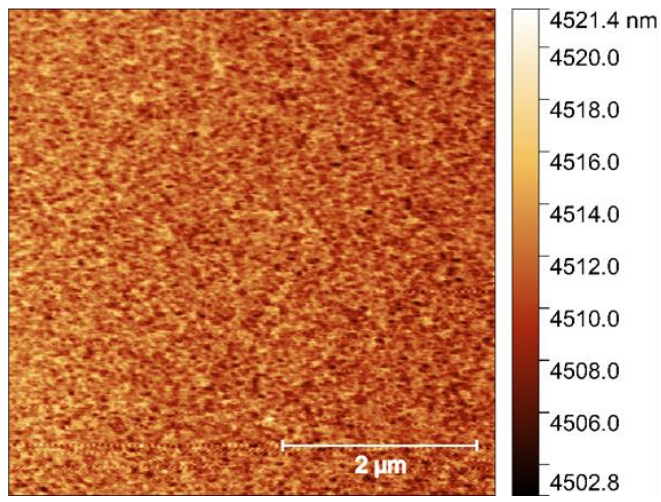

(B)

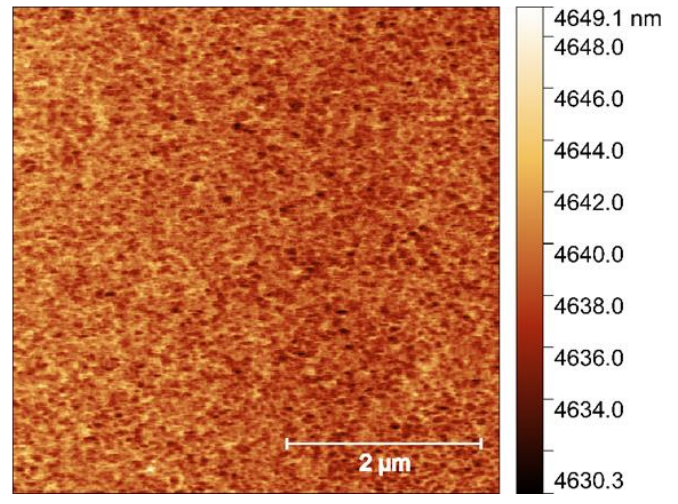

Figure S5. The FM-AFM images of the thin-film of the P3CPT surface with the HEPES buffer solution $(100 \mathrm{mM})$ containing $\mathrm{NaCl}(100 \mathrm{mM})$ in A) the absence or B) the presence of the $\mathrm{Cu}^{2+}$ ion $(40 \mu \mathrm{M})$ at $\mathrm{pH}$ 7.4. Each solution $(50 \mu \mathrm{L})$ was dropped on the channel region of the WG-OFET. The AFM measurement was carried out after immersion of the device in the aqueous solution at least $2 \mathrm{~h}$.

\section{The WG-OFET characteristics related with the sensing application}

Table S1. Response time of $\mathrm{Cu}^{2+}$ in each concentration.

\begin{tabular}{cc}
\hline $\mathrm{Cu}^{2+}$ concentration $(\mu \mathrm{M})$ & Saturation time $(\mathrm{s})$ \\
\hline 1 & $2.3 \times 10^{3}$ \\
3 & $1.2 \times 10^{3}$ \\
5 & $8.9 \times 10^{2}$ \\
10 & $3.4 \times 10^{2}$ \\
\hline
\end{tabular}




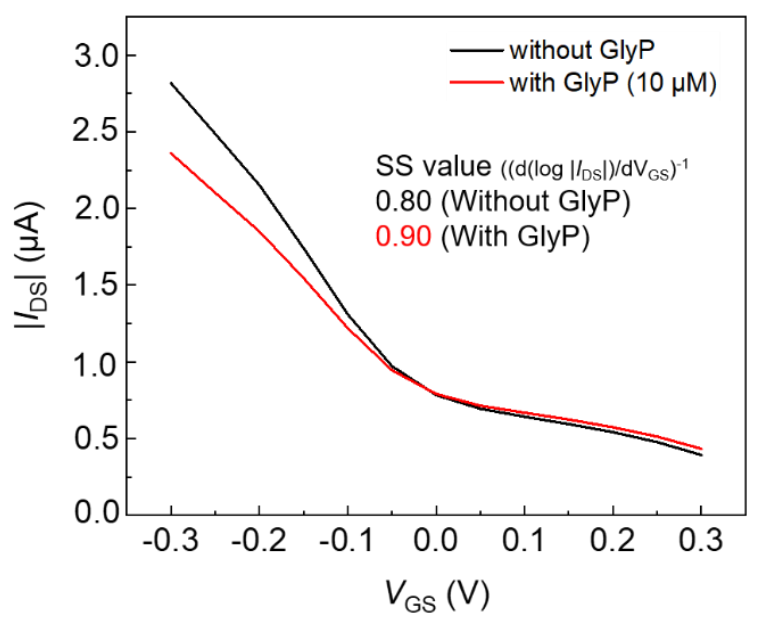

Figure S6. Transfer characteristics of the WG-OFET at a static condition in the presence (red line) or absence (black line) of GlyP $(10 \mu \mathrm{M})$. The SS values were estimated at $V_{\mathrm{DS}}=-0.2 \mathrm{~V}, V_{\mathrm{GS}}=-0.3 \mathrm{~V}$. $\left[\mathrm{Cu}^{2+}\right]$ $=40 \mu \mathrm{M}$.

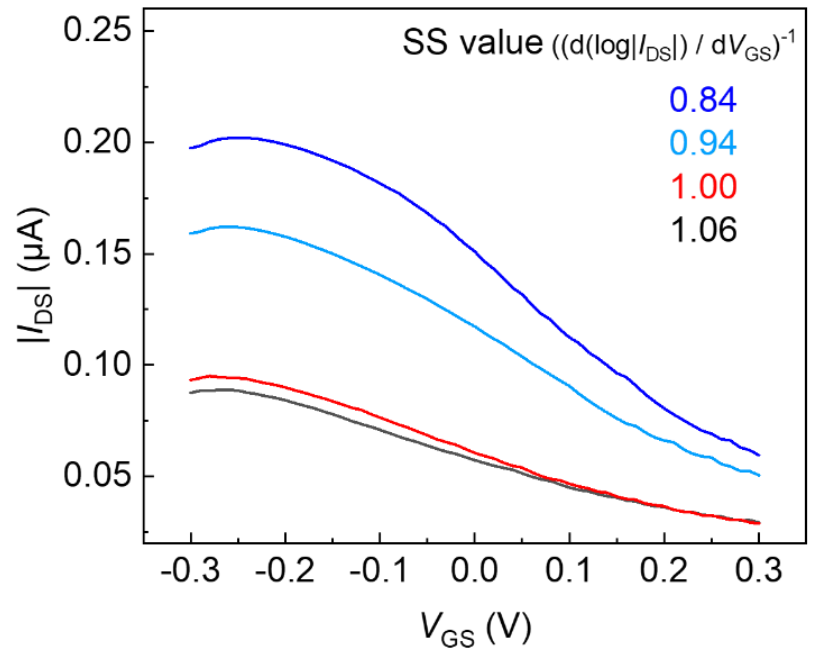

Figure S7. Transfer characteristics of the WG-OFET with the injection of the HEPES (100 $\mathrm{mM})$ buffer containing $\mathrm{NaCl}(100 \mathrm{mM})$ at a flow rate of $10 \mu \mathrm{L}$ min-1. Black line: HEPES buffer, light blue line: $\left[\mathrm{Cu}^{2+}\right]$ $=3 \mu \mathrm{M}$, blue line: $\left[\mathrm{Cu}^{2+}\right]=5 \mu \mathrm{M}$, red line: $\left[\mathrm{Cu}^{2+}\right]=5 \mu \mathrm{M},[\mathrm{GlyP}]=10 \mu \mathrm{M}$. The SS values were estimated at $V_{\mathrm{DS}}=-0.3 \mathrm{~V}, V_{\mathrm{GS}}=0 \mathrm{~V}$. 
Table S2. Response time of GlyP in each concentration. ${ }^{a}$

\begin{tabular}{cc}
\hline GlyP concentration $(\mu \mathrm{M})$ & Saturation time $(\mathrm{s})$ \\
\hline 1 & $3.8 \times 10^{2}$ \\
3 & $7.2 \times 10^{2}$ \\
5 & $9.2 \times 10^{2}$ \\
7 & $1.0 \times 10^{3}$ \\
10 & $1.5 \times 10^{3}$
\end{tabular}

${ }^{a} \mathrm{The} \mathrm{Cu}^{2+}$ solution $(5 \mu \mathrm{M})$ without GlyP flowed for 15 min before the GlyP injection.

Table S3. Comparison of sensitivity in the reported GlyP sensors.

\begin{tabular}{|c|c|c|}
\hline Type of GlyP sensor & $\operatorname{LoD}(\mu \mathrm{M})$ & Reference \\
\hline WG-OFET based sensor & 0.13 & $\begin{array}{l}\text { This study (estimated by the continuous } \\
\text { measurement) }\end{array}$ \\
\hline Electrochemical sensor & 0.003 & ChemElectroChem 2020, 7, 1558. \\
\hline Fluorescent sensor & 4.0 & Talanta 2016, 161, 94. \\
\hline Fluorescent sensor & 2.0 & J. Am. Chem. Soc. 2014, 136, 11396. \\
\hline Colorimetric sensor & 0.15 & Analyst 2019, 144, 2017. \\
\hline Colorimetric sensor & 1.0 & Sens. Actuators, B 2016, 228, 410. \\
\hline
\end{tabular}

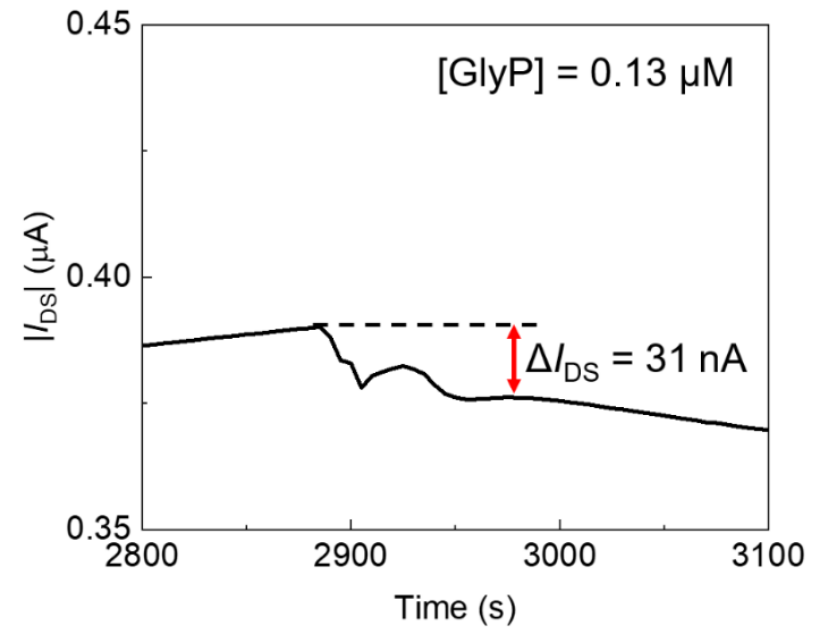

Figure S8. The real-time monitoring of GlyP at the LoD concentration with a continuous flow (10 $\mu \mathrm{L}$ $\min ^{-1}$ ) of the HEPES buffer. $\left[\mathrm{Cu}^{2+}\right]=5 \mu \mathrm{M}$, [GlyP $]=0.13 \mu \mathrm{M}$. 